\title{
Posterior Vertebrectomy and Circumferential Fusion for the Treatment of Advanced Thoracolumbar Kümmell Disease with Neurologic Deficit
}

\author{
Yongjae Cho \\ Department of Neurosurgery, Ewha Womans University School of Medicine, Seoul, Korea
}

\begin{abstract}
Study Design: Single-center, retrospective case series.
Purpose: To investigate the effectiveness of posterior vertebrectomy and circumferential fusion in patients with advanced Kümmell disease with neurologic deficit.

Overview of Literature: Various surgical options exist for the treatment of Kümmell disease, and determination of the appropriate treatment is based on the clinical and radiologic status of the patient. However, surgical intervention is required for patients with advanced Kümmell disease accompanied by neurologic deficit.

Methods: We retrospectively analyzed 22 neurologically compromised patients with advanced Kümmell disease who were treated surgically at Ewha Womans Hospital between January 2011 and January 2014. The surgical approach used by us was a posterior vertebrectomy with mesh cage insertion and segmental cement-augmented pedicle screw fixation. The tissue from the corpectomy was histopathologically examined. Anterior vertebral height, kyphotic angle, visual analog scale (VAS) score, and the Frankel classification were used to evaluate the efficacy of the procedure.

Results: The mean follow-up period was 26 months (range, 13-40 months). VAS score, anterior vertebral height, kyphotic angle, and neurologic state were significantly improved immediately postoperatively and at the last follow-up compared with preoperatively $(p<0.05)$. Most patients exhibited intravertebral clefts on imaging, and postoperative pathology revealed bone necrosis.

Conclusions: Posterior vertebrectomy with mesh cage insertion and segmental cement-augmented pedicle screw fixation is an effective approach for treating patients with advanced Kümmell disease with neurologic deficit.
\end{abstract}

Keywords: Osteoporosis; Vertebrectomy; Kyphosis

\section{Introduction}

Delayed post-traumatic vertebral collapse, or Kümmell disease, is marked by a painful kyphotic deformity that develops after an asymptomatic period of several weeks or months following an injury; it was first described by
Hermann Kümmell in 1895 [1]. Symptoms may be absent for months to years after a minor trauma, but they can develop insidiously or suddenly. Clinically, Kümmell disease is characterized by postural deformity accompanied by extreme pain and a vacuum phenomenon in the vertebral body or intervertebral disc that may be radiographically

Received Dec 5, 2016; Revised Jan 18, 2017; Accepted Jan 20, 2017

Corresponding author: Yongjae Cho

Department of Neurosurgery, Ewha Womans University Mok-Dong Hospital, Ewha Womans University School of Medicine,

1071 Anyangcheon-ro, Yangcheon-gu, Seoul 07985, Korea

Tel: +82-2-2650-2658, Fax:+82-2-2655-2652, E-mail: yongcho@ewha.ac.kr 
observed [2]. In addition to the simple radiologic findings, abnormal findings on computed tomography (CT) and magnetic resonance imaging (MRI) have been reported [3]; however, the clinical course and pathogenesis of Kümmell disease are controversial [4]. The disease has distinct developmental phases [5]. In the initial phase, patients experience backache and tenderness, which subside and are followed by an asymptomatic period. In the late phase, the pain recurs weeks to months after the initial injury without further apparent trauma, and patients develop a progressive angular kyphosis with or without neurologic deficit.

Typical radiologic findings of Kümmell disease are an intravertebral cleft (intravertebral vacuum or fluid collection) combined with a collapsed vertebra (vertebra plana) [6]. More recently, multiple synonymous terms have been used to describe Kümmell disease, including delayed posttraumatic vertebral collapse, vertebral osteonecrosis, and intravertebral pseudarthrosis [7]. Whether the presence of an intravertebral cleft is a pathognomonic sign of Kümmell disease remains controversial [4]. Kümmell disease most commonly develops at the thoracolumbar spine, which is the junctional area of the whole spine. Various treatments based on the clinical radiologic status have been introduced $[4,7,8]$.

In this study, wereport our findings on the use of vertebral column resection with mesh cage insertion, followed by polymethylmethacrylate (PMMA)-augmented pedicle screw fixation performed at one or two levels above and below the affected vertebrae to treat patients with advanced Kümmell disease with neurologic deficit. Based on a review of the literature combined with our findings, we also discuss whether an intravertebral cleft is a pathognomonic sign of Kümmell disease.

\section{Materials and Methods}

\section{Patients}

We retrospectively analyzed 22 patients ( 7 males and 15 females with a mean age of 72 years; age range, 68-83 years) admitted to our hospital with advanced thoracolumbar Kümmell diseaseand surgically treated between January 2011 and January 2014. These patients were considered to have an advanced disease because of their neurologically compromised state caused by severe vertebral collapse. The duration of symptoms in all patients was
7-12 weeks, and 18 of the patients had a definitive history of minor trauma (Table 1). The mean bone mineral density level was $-3.60 \pm 7.6$, and the collapsed vertebrae were T10 (n=2), T11 ( $=6)$, T12 ( $=10)$, L1 $(n=3)$, and L2 $(n=1)$. To assess the effectiveness of the procedure, we compared the amount of reduction in kyphotic deformity, clinical symptoms, and neurologic deficit preoperatively and postoperatively. For all patients, radiologic studies were conducted including standing anteroposterior and lateral radiographs as well as CT and MRI. The study was conducted after obtaining approval from the ethics committee of Ewha Womans Hospital. The Institutional Review Board in Ewha Womans Hospital approved the study.

\section{Surgical procedure}

The patients were placed in a prone position onto the frame to avoid compression of the intra-abdominal vessels and to decrease thoracolumbar kyphosis angle. This postural reduction restored most of the body height of the fractured vertebrae. Using a standard posterior midline approach, pedicle screws were placed promptly into the vertebrae one or two levels above and below the collapsed vertebra. Prior to the insertion of each screw, 1 or $2 \mathrm{~mL}$ of PMMA was instilled under fluoroscopic guidance into the vertebral bodies through a tapping hole that was intended for screw fixation. The affected vertebral laminae, bilateral facets, pedicles, and fractured body with the superior and inferior discs were resected to decompress the spinal cord as much as possible.

A mesh cage packed with autologous bone chip was inserted into the corpectomy site. One nerve root, mainly on the left side, was sacrificed as necessary to secure the space for cage insertion, and two small cages were inserted on either side of the lumbar corpectomy site. A large-diameter mesh cage or two small cages were used; this was decided intraoperatively based on the feasibility of cage insertion into the corpectomy site and the level of the affected vertebra. A temporary rod was used alternately to maintain the stability of the vertebral column during vertebrectomy and to prevent compromise of thedural sac and neural tissue. After reduction of kyphotic changes, two rods were tightened in an alternating manner. A careful inspection was performed to ensure that no compression of the dural sac, no leakage of bone cement or cerebrospinal fluid (CSF), and no bleeding occurred in the resected bony and soft tissues. Posterolateral fusion 
Table 1. Patient's demographics

\begin{tabular}{|c|c|c|c|c|c|c|c|c|c|c|c|c|c|c|}
\hline \multirow[t]{2}{*}{ No. } & \multirow[t]{2}{*}{ Sex } & \multirow[t]{2}{*}{ Age } & \multirow[t]{2}{*}{ BMD } & \multirow[t]{2}{*}{ Level } & \multirow{2}{*}{$\begin{array}{l}\text { Fusion } \\
\text { level }\end{array}$} & \multicolumn{2}{|c|}{$\begin{array}{l}\text { VAS } \\
\text { score }\end{array}$} & \multicolumn{2}{|c|}{$\begin{array}{l}\text { Kyphotic } \\
\text { angle }\left({ }^{\circ}\right)\end{array}$} & \multicolumn{2}{|c|}{$\begin{array}{c}\text { Anterior } \\
\text { vertebral } \\
\text { height }(\mathrm{mm})\end{array}$} & \multicolumn{2}{|c|}{$\begin{array}{c}\text { Frankel clas- } \\
\text { sification }\end{array}$} & \multirow{2}{*}{$\begin{array}{l}\text { Compli- } \\
\text { cation }\end{array}$} \\
\hline & & & & & & Pre & $\mathrm{F} / \mathrm{U}$ & Pre & $\mathrm{F} / \mathrm{U}$ & Pre & $\mathrm{F} / \mathrm{U}$ & Pre & $\mathrm{F} / \mathrm{U}$ & \\
\hline 1 & $\mathrm{~F}$ & 72 & $-3.5 \pm 8.2$ & $\mathrm{~T} 11$ & T9-L1 & 8 & 2 & 26 & 15 & 12 & 35 & C & C & \\
\hline 2 & $F$ & 75 & $-4.6 \pm 5.0$ & $\mathrm{~T} 12$ & T10-L2 & 8 & 2 & 25 & 17 & 13 & 30 & C & $E$ & \\
\hline 3 & $M$ & 78 & $-3.2 \pm 1.2$ & T11, T12 & T9-L1 & 9 & 2 & 25 & 20 & 15 & 34 & C & E & \\
\hline 4 & $M$ & 70 & $-3.9 \pm 9.7$ & $\mathrm{~T} 12$ & T10-L2 & 10 & 1 & 22 & 16 & 20 & 36 & $D$ & $E$ & CSF \\
\hline 5 & $\mathrm{~F}$ & 68 & $-3.7 \pm 2.5$ & $\mathrm{~T} 12$ & T10-L2 & 8 & 2 & 30 & 22 & 17 & 37 & $D$ & $E$ & \\
\hline 6 & M & 74 & $-4.2 \pm 4.3$ & L1 & T11-L3 & 7 & 2 & 20 & 14 & 11 & 36 & C & D & \\
\hline 7 & $F$ & 74 & $-4.0 \pm 0.2$ & $\mathrm{~T} 12$ & T10-L2 & 8 & 2 & 34 & 19 & 12 & 37 & C & $D$ & $\mathrm{Wd}$ \\
\hline 8 & $F$ & 73 & $-3.6 \pm 5.7$ & $\mathrm{~T} 12$ & T10-L2 & 8 & 2 & 40 & 35 & 14 & 31 & C & $D$ & \\
\hline 9 & M & 75 & $-3.4 \pm 1.2$ & $\mathrm{~T} 11$ & T9-L1 & 8 & 2 & 25 & 12 & 21 & 35 & D & E & \\
\hline 10 & M & 80 & $-4.9 \pm 2.4$ & $\mathrm{~T} 12$ & T10-L2 & 7 & 2 & 23 & 12 & 13 & 37 & D & $E$ & \\
\hline 11 & $\mathrm{~F}$ & 77 & $-3.2 \pm 3.1$ & $\mathrm{~T} 11$ & T9-L1 & 6 & 2 & 30 & 16 & 11 & 36 & C & E & Embol \\
\hline 12 & $F$ & 73 & $-3.7 \pm 6.2$ & $\mathrm{~T} 10$ & T10-L2 & 6 & 1 & 35 & 17 & 10 & 34 & D & $E$ & \\
\hline 13 & $\mathrm{~F}$ & 72 & $-3.0 \pm 1.0$ & $\mathrm{~T} 11$ & T9-L1 & 8 & 3 & 34 & 12 & 20 & 35 & C & $E$ & \\
\hline 14 & $\mathrm{~F}$ & 71 & $-3.2 \pm 7.2$ & L2 & T11-L3 & 5 & 3 & 33 & 22 & 17 & 36 & D & $D$ & \\
\hline 15 & M & 73 & $-3.9 \pm 8.0$ & $\mathrm{~T}, 1112$ & T10-L2 & 7 & 2 & 34 & 15 & 12 & 37 & $D$ & $E$ & \\
\hline 16 & M & 74 & $-2.8 \pm 5.0$ & $\mathrm{~T} 11$ & T9-L1 & 8 & 2 & 37 & 28 & 16 & 40 & D & E & \\
\hline 17 & $F$ & 73 & $-4.0 \pm 8.0$ & $\mathrm{~T} 11$ & T9-L1 & 8 & 3 & 40 & 36 & 14 & 35 & C & C & \\
\hline 18 & $F$ & 69 & $-3.6 \pm 3.5$ & L1 & T11-L3 & 8 & 2 & 25 & 11 & 12 & 33 & C & $D$ & \\
\hline 19 & $\mathrm{~F}$ & 68 & $-3.7 \pm 3.4$ & $\mathrm{~T} 12$ & T10-L2 & 8 & 2 & 35 & 28 & 11 & 32 & D & D & \\
\hline 20 & $\mathrm{~F}$ & 83 & $-3.4 \pm 8.6$ & $\mathrm{~T} 11$ & T9-L1 & 7 & 2 & 44 & 35 & 16 & 35 & D & $D$ & \\
\hline 21 & $\mathrm{~F}$ & 69 & $-3.8 \pm 5.3$ & $\mathrm{~T} 12, \mathrm{~L} 1$ & T11-L3 & 6 & 1 & 35 & 15 & 22 & 38 & C & E & \\
\hline 22 & $\mathrm{~F}$ & 72 & $-3.9 \pm 7.2$ & $\mathrm{~T} 12$ & T10-L2 & 6 & 3 & 30 & 14 & 20 & 35 & C & $E$ & \\
\hline
\end{tabular}

BMD, bone mineral density (Dual-energy X-ray absorptiometry); VAS, visual analog scale; Pre, preoperative; F/U, last follow-up; F, female; M, male. CSF, cerebrospinal fluid leakage; Wd, wound infection; Embol, pulmonary embolism.

with autogenous bone grafts from the resected bone was performed on the secured vertebral laminae, facets, and transverse laminae (Fig. 1). Patients were allowed to walk as early as possible while wearing braces.

\section{Clinical and radiologic evaluation}

Postoperative evaluation was performed immediately postoperatively; at 1, 3, and 6 months; and at 1 and 2 years. Anterior vertebral height was measured along the borders of the anterior vertebral body. The Cobb angle used to assess kyphosis was measured as the subtended angle between the superior endplate of the vertebra one level above the fractured vertebra and the inferior endplate of the vertebra one level below the fractured verte- bra. The presence of an intradiscal or intravertebral band of gas on plain radiography, CT, and/or MRI was considered to be an intravertebral cleft sign. Even if a cleft sign was observed in only one of the three imaging modalities, the cleft sign was concluded to be present. The visual analog scale (VAS), which ranges from 0 (no pain) to 10 (maximal pain), was used to assess pain severity, and the Frankel classification was used to assess the neurologic status pre- and postoperatively [9].

\section{Statistical analysis}

SPSS ver. 12.0 statistical software (SPSS Inc., Chicago, IL, USA) was used for data analysis. Data are presented as mean \pm standard deviation. Student's $t$-test was used to 

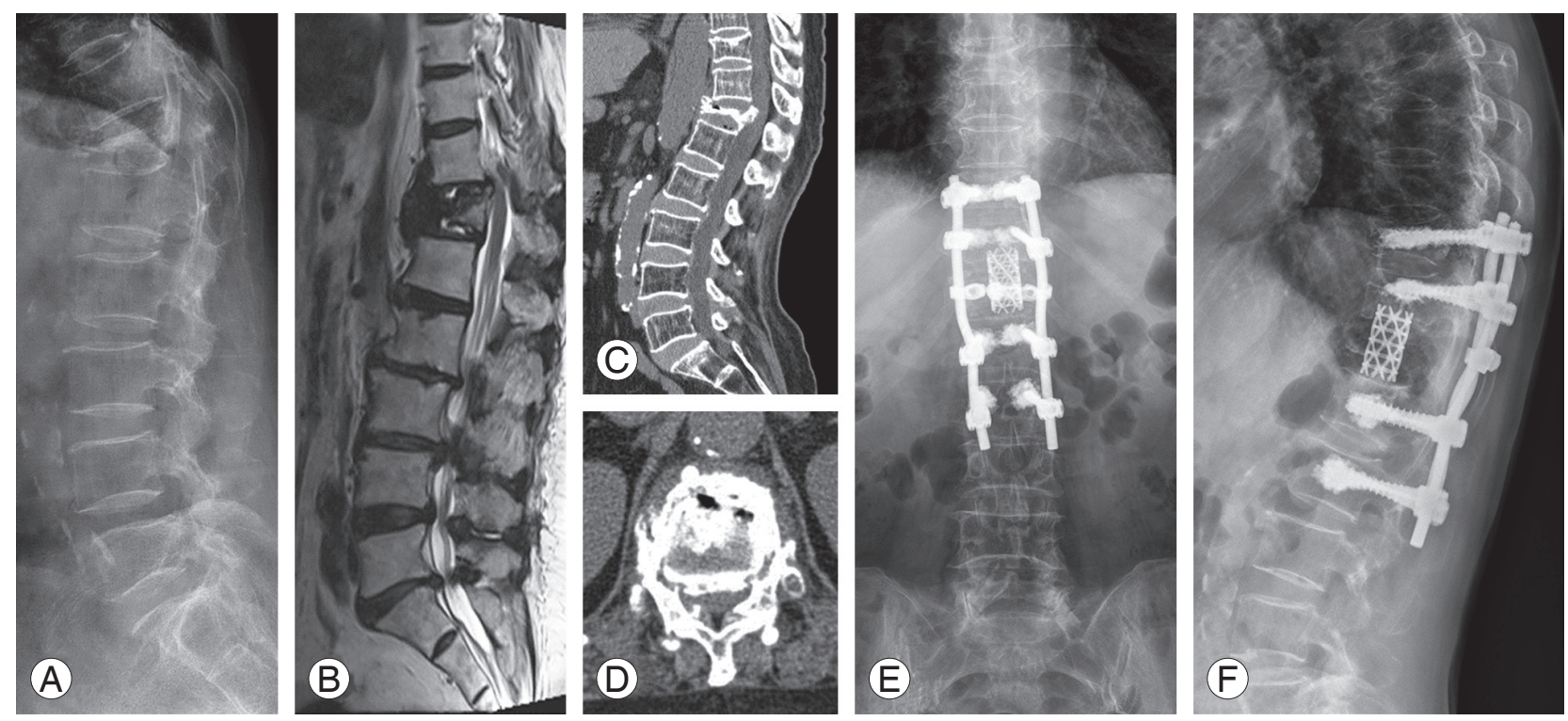

Fig. 1. (A-D) Preoperative images. (E, F) Postoperative images. (A) Preoperative standing lateral radiograph revealed a collapsed fracture at T12 and kyphosis with an intravertebral cleft. (B) Preoperative magnetic resonance imagedisplayed mixed signs of liquid and gas within the vertebral body. (C, D) Preoperative computed tomography image showed the intravertebral vacuum sign and gas in the adjacent disc. (E, F) Lateral radiographs showed no decrease in the vertebral height orrecurrence of kyphosis at 2-year follow-up.

evaluate the significance of changes in VAS scores, kyphotic angles, and vertebral body heights preoperatively, immediately postoperatively, and at the final follow-up. A $p<0.05$ was considered to be significant.

\section{Results}

Of the 22 patients, 15 presented with an intravertebral cleft sign at the initial radiologic examination; intravertebral (10 patients), intradiscal (5 patients), and both intravertebral and intradiscal patterns (2 patients) were observed. Cleft signs were observed on plain radiographsin 8 cases, CT images in 15 cases, and MRI images in 8 cases. The mean duration of surgery was 201 minutes (range, 180-250 minutes), and the mean estimated blood loss was $550 \mathrm{~mL}$ (range, $340-800 \mathrm{~mL}$ ). The mean volume of PMMA used at one screw level was $2.6 \mathrm{~mL}$ (range, 2.0-3.5 $\mathrm{mL}$ ). A spinal dural tear occurred in one patient. Intraop- erative biopsies from 16 patients revealed bone necrosis, while the remaining 6 patients had bone fracture. Fusion levels were T10-L2 (10 patients), T9-L1 (8 patients), and T11-L3 (4 patients). There were no neurologic deficits, despite sacrifice of the nerve root (Table 1).

Patients were followed-up for 13-40 months (mean, 26 months). Mean VAS score, anterior height of the affected vertebrae, and kyphotic angle improved significantly immediately postoperatively compared with the preoperative state $(p<0.05)$. These improvements were maintained from immediately postoperatively till the final follow-up $(p<0.05)$ (Table 2). None of the patients was classified as grade A under the Frankel classification. Preoperatively, 12 patients were classified as grade $\mathrm{C}$ and 10 as grade $\mathrm{D}$; immediately postoperatively, 10 patients were classified as grade C, 6 as grade D, and 6 as grade E. At the final follow-up, 2 patients were classified as grade C, 7 as grade $\mathrm{D}$, and 13 as grade $\mathrm{E}$. None of the patients deteriorated to

Table 2. Analysis prior and following surgery

\begin{tabular}{lccc} 
& Kyphotic angle $\left(^{\circ}\right)$ & Anterior vertebral height $(\mathrm{mm})$ & VAS \\
Preoperative & $31.95 \pm 44$ & $13.22 \pm 7.8$ & $8.59 \pm 0.41$ \\
Immediate postoperative & $15.24 \pm 7.8$ & $35.10 \pm 9.6$ & $2.09 \pm 0.35$ \\
\hline Last follow-up & $19.56 \pm 8.6$ & $31.54 \pm 7.0$ & $2.78 \pm 0.95$ \\
\hline
\end{tabular}

VAS, visual analog scale.

$p<0.05$; Preoperative values vs. immediate postoperative, Preoperative values vs. last follow-up. 
a worse neurologic status postoperatively compared with their preoperative status. Furthermore, the neurologic functioning of all the patients improved by one or two levels at the final follow-up (Table 1). Some superficial complications were observed: skin infection (one patient), delayed CSF leakage (one patient), and pulmonary pneumonia (one patient). No obvious loosening of internal fixation, breakage, or bone cement displacement occurred during follow-up.

\section{Discussion}

Since Maldague et al. [10] first reported the intravertebral vacuum cleft (described as gas accumulation in the vertebral body visible on plain radiograph), it has been considered a pathognomonic sign of Kümmell disease. The vacuum phenomenon is more evident with the spine in the extended position and may be reduced or disappear in the flexed position [11]. In our study, the intravertebral cleft was more readily observed using CT than usingt he other imaging modalities; this may be due to the relatively high resolution of CT for visualizing gas and bone. However, whether the intravertebral cleft is a pathognomonic sign of Kümmell disease remains controversial [12]. It has been recommended that the presence of an intravertebral cleft should not necessarily be considered as a characteristic a sign of Kümmell disease, but if the cleft is present, the disease should be suspected [12]. Therefore, we considered it necessary to confirm the presence of Kümmell disease by performing biopsy to evaluate the presence of bone necrosis. The pathogenesis of the intravertebral vacuum phenomenon remains controversial. It is hypothesized to be related to vertebral avascular necrosis, vertebral fracture nonunion, pseudarthrosis, or intradiscal gas leakage through the endplate fractured into the vertebral body [13].

Most patients with early-stage Kümmell disease have no real neurologic deficits, but they experience severe pain during motion. These patients are generally treated with bed rest and analgesic anti-inflammatory drugs [14]. However, these may not be the most appropriate methods of treatment if the pain is chronic, does not respond well to conservative treatment, and persists and long-term bed rest cannot be maintained [14]. Furthermore, it is important to determine whether the pain originates from a compression fracture, is caused by a kyphotic deformity, or results from fractures related to other spinal lesions (spinal stenosis, disc herniation, etc.). The treatment method should be determined according to the patient's neurologic and systemic status, radiologic findings, and the cause of pain and kyphosis [15].

Treatment strategies for Kümmell disease differ according to the clinical course of the disease. For patients having pain without neurologic symptoms, the objective is to eliminate pain at the fracture site and restore stability. Some authors have reported that percutaneous vertebroplasty or kyphoplasty yields good outcomes in patients who have persistent pain without neurologic symptoms $[7,14]$. The mechanism by which vertebroplasty alleviates pain is unclear, but itmay involve thermal necrosis, lysis of nerve cell endings due to chemical toxicity, and improvement of mechanical stability [16].

Some researchers have advocated the use of cement instillation within collapsed vertebral bodies in patients with Kümmell disease [17]. In patients with advanced disease, vertebroplasty or kyphoplasty are unsuitable and may further worsen the neurologic status [18]. Various surgical methods, such as anterior decompression and fusion with bone graft, posterior decompression with pedicle subtraction osteotomy, and a combined anterior and posterior approach have been introduced, but these procedures have not led to satisfactory outcomes; rather, instrument failure, progression of kyphosis, delayed recovery of the neurologic deficit, and failure of early ambulation have been reported with these techniques [5]. For neurologically impaired patients, the aim of surgery is to decompress the spinal cord, restore the physiologic spinal curvature, and maintain spinal stability.

Surgical options for advanced Kümmell disease include anterior, posterior, or combined anterior and posterior approaches. For example, anterior decompression and fusion with an intervertebral tricortical graft or mesh cage have yielded favorable outcomes. These procedures are most efficient for decompressing the spinal cord because the focus of pathology, which is an incompetent as well as compressed vertebral column, can be directly accessed. In addition, an anterior column support is provided, resulting in a relatively high fusion rate and a favorable postoperative kyphosis correction angle. However, the disadvantage of the anterior approach in retroperitoneal, extrapleural surgical procedures is that it may cause pulmonary, urologic, intra-abdominal, and/or retroperitoneal complications. In addition, most cases of Kümmell disease occur in elderly individuals who may have several car- 
diopulmonary abnormalities. Moreover, graft collapse or fusion failure may occur due to osteoporotic bone when using the anterior approach alone [5].

The posterior approach alone involves decompression, resection of the fractured vertebra, and fusion. The fusion rate achieved with this approach is relatively high, and immediate stability is obtained. However, because of the lack of an anterior support and decompression, the longterm failure rate is high, and incomplete decompression has been reported. A combined anterior and posterior approach results in a favorable fusion rate with correction of kyphosis over the short and long term. However, considering the elderly nature of the patients, surgery time is long, and a large amount of blood are significant drawback [5].

Because patients with advanced Kümmell disease with neurologic symptoms are often elderly and have a variety of comorbidities, they do not easily tolerate multiple surgical procedures. Consequently, the development of a single-stage treatment method is required. Surgeons have performed single-stage posterior decompression and segmental fixation in patients with advanced Kümmell disease and neurologic symptoms. The decompression and segmental fixation are followed by anterior cage insertion and bone cement-augmented screw fixation under direct visualization. This procedure has several advantages. Posterior corpectomy with mesh cage insertion relieves cord compression, and augmented segmental fixation and fusion reduce the number of fusion segments and the influence of long segmental spinal function. Bone cement in the vertebral body allows a firm integration of screws and provides anterior support to minimize posterior pedicle screw stress.

We followed our patients for a mean duration of 26 months. At the final follow-up, slight decreases in vertebral height and kyphosis correction angle were observed compared with those measured 1 day postoperatively. However, these differences were not significant. All the patients had severe osteoporosis, which may have resulted in the loss of correction observed during follow-up. The neurologic status of our patients had improved by at least one Frankel grade at the final follow-up, and most of the patients were ambulatory. Thus, we conclude that posterior vertebrectomy with mesh cage insertion and segmental cement-augmented pedicle screw fixation for circumferential fusion is an effective treatment for patients with Kümmell disease with neurologic compromise.
Our study had some limitations. First, recruitment bias was inevitable and may have affected our findings. The small number of subjects and the relatively short followup durations are additional limitations. Furthermore, this was a case series study without a control group. To better evaluate the surgical outcomes, case-controlled studies are needed.

Ours was a preliminary study, and we recommend that future studies on Kümmell disease should be carefully designed for further clinical interpretation. A larger number of patients and longer follow-up periods are required to verify the efficacy of our approach for treating advanced Kümmell disease with neurologic deficit.

\section{Conclusions}

We retrospectively analyzed the outcomes in 22 neurologically compromised patients with advanced Kümmell disease who underwent vertebrectomy with mesh cage insertion and segmental PMMA-augmented pedicle screw fixation. Outcomes were favorable, and we, therefore, consider this surgical approach to be a viable option for the treatment of advanced Kümmell disease with neurologic deficit.

\section{Conflict of Interest}

No potential conflict of interest relevant to this article was reported.

\section{References}

1. Nickell LT, Schucany WG, Opatowsky MJ. Kummell disease. Proc (BaylUniv MedCent) 2013;26:300-1.

2. Linn J, Birkenmaier C, Hoffmann RT, Reiser M, Baur-Melnyk A. The intravertebral cleft in acute osteoporotic fractures: fluid in magnetic resonance imaging-vacuum in computed tomography? Spine (Phila Pa 1976) 2009;34:E88-93.

3. Oka M, Matsusako M, Kobayashi N, Uemura A, Numaguchi Y. Intravertebral cleft sign on fatsuppressed contrast-enhanced MR: correlation with cement distribution pattern on percutaneous vertebroplasty. Acad Radiol 2005;12:992-9.

4. Wu AM, Ni WF, Weng W, Chi YL, Xu HZ, Wang XY. Outcomes of percutaneous kyphoplasty in patients with intravertebral vacuum cleft. Acta Orthop Belg 
2012;78:790-5.

5. Wu AM, Chi YL, Ni WF. Vertebral compression fracture with intravertebral vacuum cleft sign: pathogenesis, image, and surgical intervention. Asian Spine J 2013;7:148-55.

6. Osterhouse MD, Kettner NW. Delayed posttraumatic vertebral collapse with intravertebral vacuum cleft. J Manipulative Physiol Ther 2002;25:270-5.

7. Zhang X, Hu W, Yu J, Wang Z, Wang Y. An Effective treatment option for Kummell disease with neurological deficits: modified transpedicular subtraction and disc osteotomy combined with long-segment fixation. Spine (Phila Pa 1976) 2016;41:E923-30.

8. Nakamae T, Fujimoto Y, Yamada K, Hashimoto T, Olmarker K. Efficacy of percutaneous vertebroplasty in the treatment of osteoporotic vertebral compression fractures with intravertebral cleft. Open Orthop J 2015;9:107-13.

9. Capaul M, Zollinger H, Satz N, Dietz V, Lehmann D, Schurch B. Analyses of 94consecutive spinal cord injury patients using ASIA definition and modified Frankel score classification. Paraplegia 1994;32:5837.

10. Maldague BE, Noel HM, Malghem JJ. The intravertebral vacuum cleft: a sign of ischemic vertebral collapse. Radiology1978;129:23-9.

11. Malghem J, Maldague B, Labaisse MA, et al. Intravertebral vacuum cleft: changes in content after supine positioning. Radiology1993;187:483-7.
12. Hatano H, Oike N, Ariizumi T, Sasaki T, Kawashima $\mathrm{H}$. Intravertebral cleft in pathological vertebral collapse resulting from cancer metastasis: report of three cases. Skeletal Radiol 2016;45:1747-50.

13. Kawaguchi S, Horigome K, Yajima H, et al. Symptomatic relevance of intravertebral cleft in patients with osteoporotic vertebral fracture. J Neurosurg Spine 2010;13:267-75.

14. Chen GD, Lu Q, Wang GL, et al. Percutaneous kyphoplasty for Kummell disease with severe spinal canal stenosis. Pain Physician 2015;18:E1021-8.

15. Park YS, Kim HS. Prevention and treatment of multiple osteoporotic compression fracture. Asian Spine J 2014;8:382-90.

16. Kim SB, Jeon TS, Lee WS, Roh JY, Kim JY, Park WK. Comparison of kyphoplasty and lordoplasty in the treatment of osteoporotic vertebral compression fracture. Asian Spine J 2010;4:102-8.

17. Huang Y, Peng M, He S, Tang X, Dai M, Tang C. Clinical efficacy of percutaneous kyphoplasty at the hyperextension position for the treatment of osteoporotic Kummell disease. Clin Spine Surg2016;29:1616.

18. Jung JY, Lee MH, Ahn JM. Leakage of polymethylmethacrylate in percutaneous vertebroplasty: comparison of osteoporotic vertebral compression fractures with and without an intravertebral vacuum cleft. J Comput Assist Tomogr 2006;30:501-6. 